\title{
Evaluation des performances agronomiques de quinze nouvelles lignées de tomate en station au centre de la Côte d'Ivoire
}

\author{
Noupé Diakaria COULIBALY ${ }^{1 *}$, Lassina FONDIO ${ }^{1}$, Mako FDP N'GBESSO $^{1}$ et \\ Bakary DOUMBIA ${ }^{2}$ \\ ${ }^{1}$ CNRA (Centre National de Recherche Agronomique), 01 BP 633 Bouaké 01, Côte d'Ivoire. \\ ${ }^{2}$ Université Jean Lorougnon Guédé de Daloa, BP 150 Daloa, Côte d'Ivoire. \\ *Auteur correspondant; E-mail: noupediakaria@gmail.com; Tel: (+225) 07633239
}

\section{RESUME}

Une étude a été réalisée à la station de recherche sur les cultures vivrières du CNRA de Bouaké en vue de déterminer les performances agronomiques de quinze (15) nouvelles lignées de tomate. Ces lignées ont été évaluées, selon un dispositif en blocs de Fisher avec trois répétitions. Les observations et mesures ont porté en autres sur le développement végétatif, les stades phénologiques et le rendement. Les résultats obtenus ont révélé une variabilité entre les lignées pour l'état végétatif et la hauteur des plants. La taille des plants a varié entre 41 et $62 \mathrm{~cm}$. La plus grande taille a été produite par la lignée TM41/14EW. L'étude de la phénologie a montré que le délai de floraison a varié entre 52 et 63 jours après le semis (JAS) et celui de la première récolte a été compris entre 96 et 101 JAS. Pour ces paramètres, TM39/14EW, TM47/14EW et TM48/15BF ont été tardives. L'évaluation des rendements a permis de noter que les lignées TM44/14EW, TM40/14EW, TM43/4EW et TM42/14EW ont été les plus productives avec un rendement moyen de plus de 30 t/ha. Les lignées TM40/14EW et TM43/14EW ont produit des nombres élevés de fruits par plant et qui ont été les plus fermes. A l'issue de cette étude, il est à noter que les objectifs ont été atteints car des lignées à hauts rendements et à fruits fermes s'adaptant bien aux conditions climatiques de la Côte d'Ivoire ont été identifiées. En conséquence, la confirmation en milieu paysan de lignées telles que TM40/14EW, TM43/14EW et TM44/14EW devrait être envisagée.

(C) 2019 International Formulae Group. All rights reserved

Mots clés: Tomate, lignée, rendement, fermeté, Côte d'Ivoire.

\section{Evaluation of the agronomic performances of fifteen new tomato lines at the station in central Côte d'Ivoire}

\begin{abstract}
A study was conducted at the research station on food crops CNRA Bouake to determine the agronomic performance of fifteen (15) new tomato lines. These lines were evaluated according to a Fisher block device with three repetitions. Observations and measurements included vegetative development, phenological stages and yield. The results obtained revealed a variability between the lines for the vegetative state and the height of the plants. The size of the plants varied between 41 and $62 \mathrm{~cm}$. The largest size was produced by the TM41/14EW line. The study of phenology showed that the flowering time varied between 52 and 63 days after sowing (DAS) and the first harvest was between 96 and 101 DAS. For these parameters,
\end{abstract}


TM39/14EW, TM47/14EW and TM48/15BF were late. The yield assessment noted that the TM44/14EW, TM40/14EW, TM43/4EW and TM42/14EW lines were the most productive with an average yield of more than 30 t/ha. The TM40/14EW and TM43/14EW lines produced high numbers of fruits per plant and were the firmest. At the end of this study, it should be noted that the objectives were achieved because high yield lines and firm fruit adapted to the climatic conditions of Côte d'Ivoire were identified. As a result, confirmation in the farm setting of lines such as TM40/14EW, TM43/14EW and TM44/14EW should be considered.

(C) 2019 International Formulae Group. All rights reserved

Keywords: Tomato, lineage, yield, firmness, Ivory Coast.

\section{INTRODUCTION}

La tomate (Solanum lycopersicum) qui est une plante annuelle de la famille des solanacées, est cultivée pour ses fruits très recherchés. Riche en éléments minéraux, lycopène, carotenoïde, vitamines $\mathrm{A}, \mathrm{C}$ et $\mathrm{E}$ (Daniel et al., 2012 ; Ignace et al., 2015) puis en antioxydants phénoliques (Martine et al., 2008). La consommation de la tomate contribue à un régime alimentaire sain et équilibré pour l'homme (Willcox et al., 2003). La tomate est cultivée dans de nombreux pays du monde et sous divers climats, y compris les régions relativement froides grâce au développement des cultures sous abri (FAO, 2007). Elle constitue, après la pomme de terre, le deuxième légume frais ou transformé, le plus consommé dans le monde (INRA, 2010). Selon cette source, la production mondiale de tomate a connu une forte croissance depuis 1978 , où elle est passée de 48 millions de tonnes à 74 millions en 1992, et à 89 millions de tonnes en 1998 pour atteindre 124 millions en 2006.

En Côte d'Ivoire, la production était estimée par la FAO en 2014 à 32364 tonnes contre un besoin de 100000 tonnes par an (Senan et al., 2007). La tomate est une denrée alimentaire importante car fortement consommée par toutes les populations du pays. En outre, au plan économique, de nombreux acteurs (producteurs et commerçants) vivent de la production et de la commercialisation de la tomate dans le pays. Cependant, plusieurs facteurs limitent la production de cette culture en Côte d'Ivoire. La principale contrainte demeure l'absence de variétés performantes adaptées aux conditions locales de production. En effet, les variétés vulgarisées ou commercialisées sont en général sensibles aux facteurs biotiques et abiotiques (Jean-Claude et al., 2019).

Pour contribuer à l'amélioration de la productivité de la tomate en Côte d'Ivoire, de nombreux travaux de recherche ont été effectués ces dernières années (Senan et al., 2007 ; N'zi et al., 2010 ; Fondio et al., 2013; Fondio et al., 2015). Toutefois, la disponibilité de variétés de tomate adaptées aux besoins des producteurs et consommateurs reste faible. De plus, la variété TMA97 vulgarisée par le Centre National de Recherche Agronomique (CNRA) rencontre des difficultés de commercialisation. En effet, de nombreux producteurs trouvent les fruits peu fermes pour supporter les conditions de transport sur de longues distances.

Le présent travail a objectif principal de sélectionner des variétés de tomate performantes caractérisées par de hauts rendements, une forte tolérance aux facteurs biotiques et abiotiques avec des fruits fermes. De façon spécifique, il s'agira d'identifier des lignées de tomate introduites productives à fruits fermes et adaptées aux zones agroécologiques de la Côte d'Ivoire.

\section{MATERIELS ET METHODES Matériel végétal}

L'essai a porté sur 15 lignées de tomates : 3 lignées du Burkina Faso, 6 lignées introduites de East West Seed Global Orion (EWSGO) en Norvège, 3 lignées originaires de Asian Vegetable Research and Development Center (AVRDC) en Taiwan, 2 variétés commerciales beaucoup cultivées par les producteurs locaux et une variété améliorée du CNRA. Ces lignées 
appartiennent à l'espèce Solanum lycopersicum (Figures 1, 2, 3 et 4). Elles ont été codifiées de la manière suivante : TM pour tomate, le chiffre suivant représente le numéro d'enregistrement dans les fichiers du CNRA, les 2 chiffres après la barre indiquent l'année d'entrée au CNRA et les lettres finales sont les initiales des lieux de provenances. Les variétés commerciales sont nommées Lindo et Petomech puis la variété du CNRA, TMA97 qui a servi de témoin dans cet essai (Tableau $1)$.

\section{Méthodes d'études \\ Dispositif expérimental et traitements}

L'essai a été disposé en blocs de Fisher à 3 répétitions. La parcelle élémentaire était composée d'une planche de $6 \mathrm{~m}^{2}(6 \mathrm{mx} 1 \mathrm{~m})$. Les plants ont été repiqués sur la planche en doubles lignes à raison de $0,5 \mathrm{~m}$ entre les lignes et $0,50 \mathrm{~m}$ sur la ligne, soit 22 plants par parcelle élémentaire. La distance entre deux parcelles élémentaires a été de $0,5 \mathrm{~m}$. Une allée de $1 \mathrm{~m}$ de large a été observée autour de la parcelle.

\section{Observations et mesures des plants}

Les observations et mesures ont été effectuées selon les différents stades de développement des cultures. L'échantillonnage a consisté à choisir au hasard dix (10) plants par parcelle élémentaire pour la mesure des paramètres agromorphologiques. Après le repiquage au champ, les observations ont porté sur les paramètres suivants:

- le nombre de plants ayant repris 15 jours après le repiquage (JAR) ;

- l'état de développement végétatif des plants. Il a été apprécié $30 \mathrm{JAR}$, suivant l'échelle de notation allant de 1 à 5 ( $1=$ très mauvais ; $2=$ mauvais ; $3=$ moyennement bon ; $4=$ bon ; $5=$ très bon) ;

- la hauteur des plants au stade de la floraison ;

- la détermination de la durée des stades phénologiques a porté sur le délai de floraison et la date de première récolte. En ce qui concerne le délai de floraison, il est noté lorsque $50 \%$ des plants de la parcelle élémentaire ont fleuri. Quant à la date de première récolte, elle est également déterminée quand la moitié des plants de la parcelle élémentaire porte des fruits mûrs ;

- les composantes du rendement ont été évaluées à partir des récoltes. Ainsi, à chaque récolte, le nombre et le poids des fruits sains et ceux avariés ont été déterminés. Ces valeurs ont permis de calculer les rendements potentiel et net et les taux de fruits sains et ceux avariés pour chaque variété ;

- La détermination du degré de fermeté du fruit de chaque lignée a été faite à la première récolte. Pour ce faire, dix (10) fruits sains de chaque lignée ont été choisis au hasard. La fermeté a été notée sur une échelle de 1 à $5 \quad(1=$ fruits complètement mous, $2=$ fruits mous, $3=$ fruits moyennement fermes, $4=$ fruits fermes, $5=$ fruits très fermes). Cette détermination de la fermeté des fruits a été faite par pression digitale.

\section{Analyses statistiques}

Toutes les données recueillies ont été analysées à l'aide du logiciel STATISTICA 7.1. Une analyse des variances (ANOVA) à un critère de classification a été réalisée pour l'ensemble des paramètres étudiés. Lorsqu'une différence significative a été observée entre les différents caractères $(\mathrm{p}<$ $0,05)$, la comparaison des moyennes a été faite à l'aide des tests LSD de Duncan et de Fisher au seuil de 5\%. Comme analyse multivariée, la classification ascendante hiérarchique (CAH) a été utilisée. Elle a permis de visualiser le degré de ressemblance entre les lignées de tomate. La méthode de Ward (1963) prise comme critère d'agglomération, la distance euclidienne, et l'indice de similarité ont été utilisées comme méthode de classification. 
N. D. COULIBALY et al. / Int. J. Biol. Chem. Sci. 13(3): 1565-1581, 2019

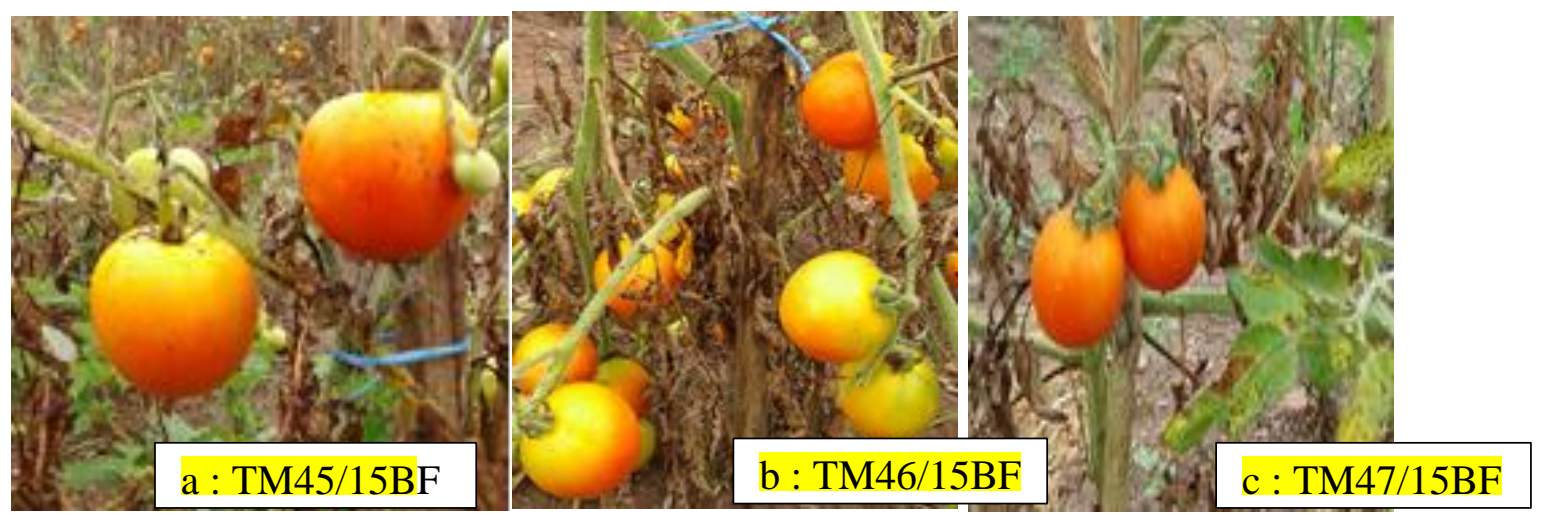

Figure 1: Les lignées en provenance du Burkina Faso.

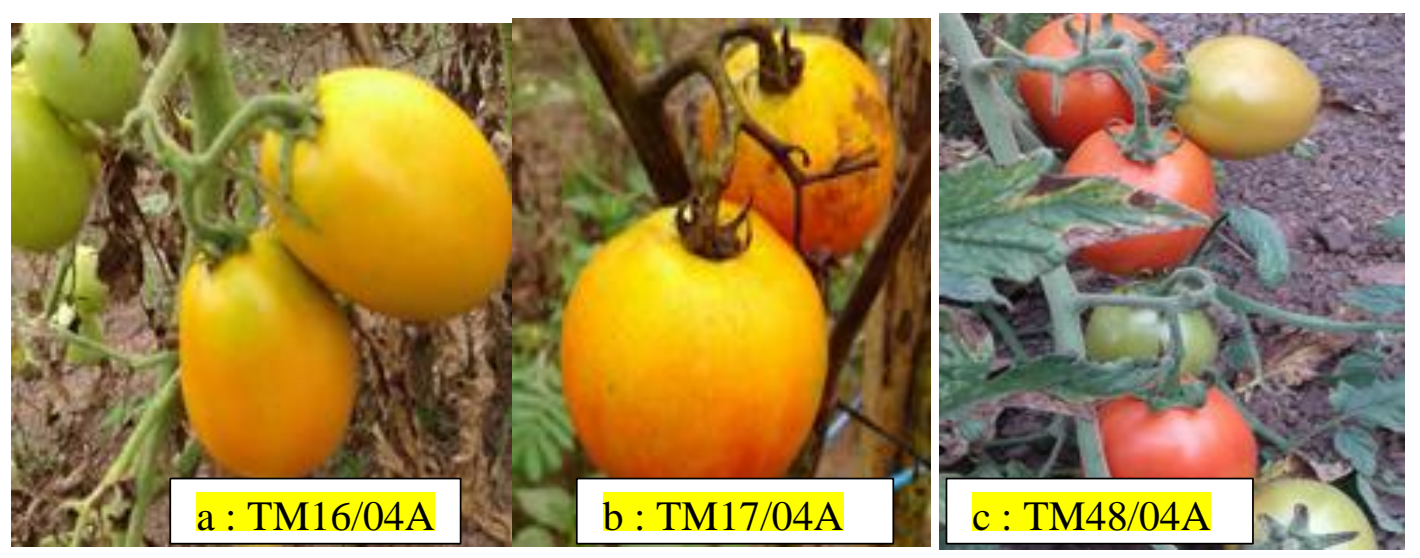

Figure 2: Les lignées venant de l'AVRDC.
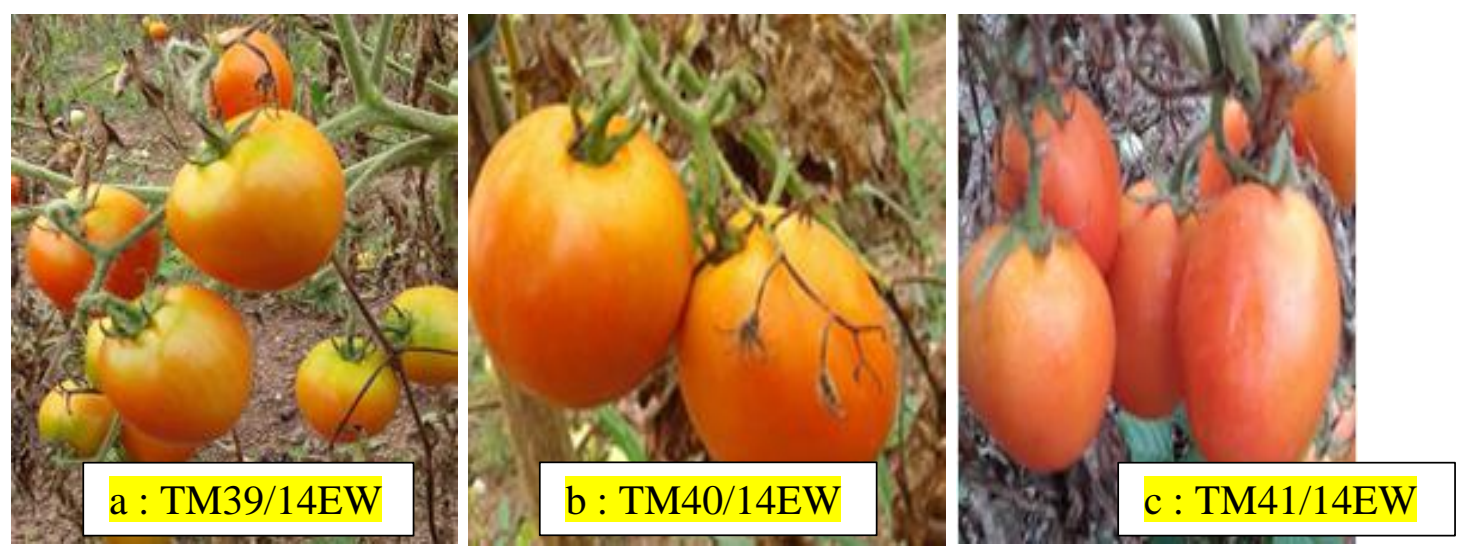

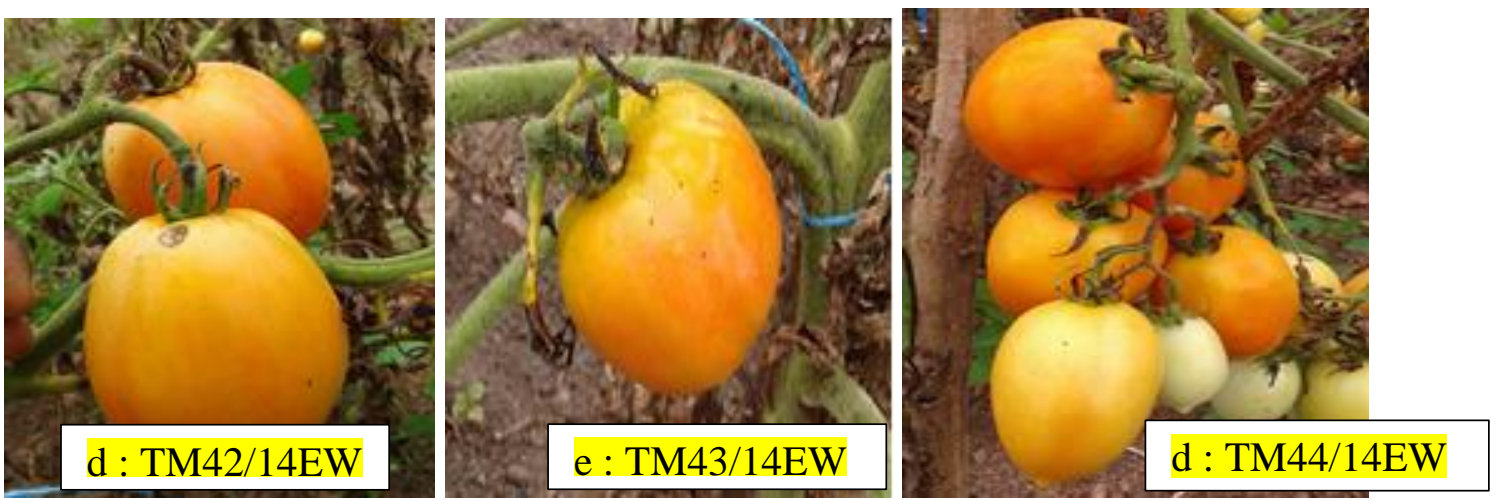

Figure 3: Les lignées issues d'EWSGO.
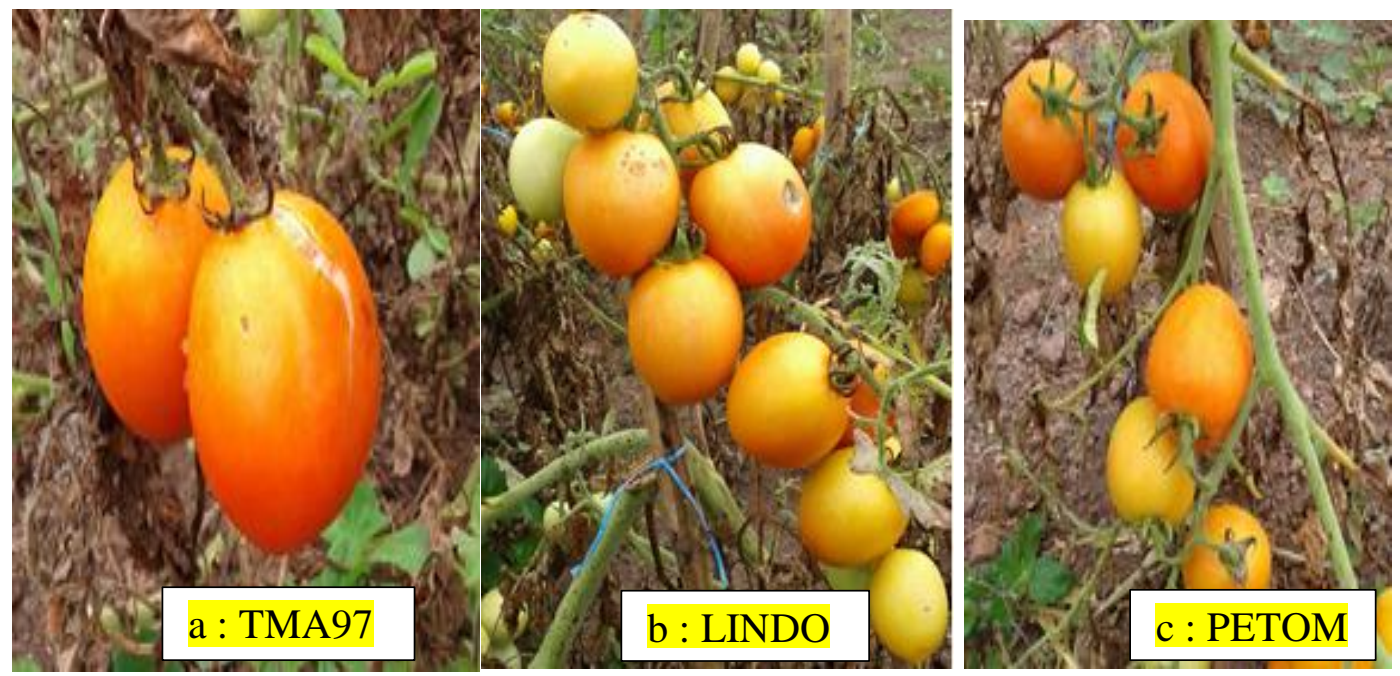

Figure 4: Les lignées de Cote d'Ivoire.

Tableau 1: Liste des lignées, leur nom d'origine, leur fournisseur et leur code d'utilisation.

\begin{tabular}{ccccll}
\hline $\mathbf{N}^{\circ}$ d'ordre & Fournisseurs & Noms d'origine & Codes CNRA & Forme du fruit & Couleur du fruit \\
\hline 1 & CNRA & TMA97 & TMA97 & Ellipsoïde & Orange \\
2 & Teechnisen & Lindo F1 & LINDO & Hautement arrondie & Jaune-orange \\
3 & Semivoire & Petomech & PETOM & Ellipsoïde & Orange \\
4 & Burkina Faso & FB T1 & TM45/15BF & Légèrement aplatie & Jaune-orange \\
5 & Burkina Faso & FB T2 & TM46/15BF & Hautement arrondie & Jaune-orange \\
6 & Burkina Faso & FB T3 & TM47/15BF & Hautement arrondie & Orange \\
7 & EWSGO & JINA & TM39/14EW & Légèrement aplatie & Orange \\
8 & EWSGO & Platinium F1 & TM40/14EW & Ronde & Orange \\
9 & EWSGO & Nivana F1 & TM41/14EW & Ellipsoïde & Rouge \\
10 & EWSGO & Jelani F1 & TM42/14EW & Très légèrement & Jaune-orange
\end{tabular}




$\begin{array}{lcccll} & & & & \text { aplatie } \\ 11 & \text { EWSGO } & \text { Makis F1 } & \text { TM43/14EW } & \begin{array}{l}\text { Cylindrique } \\ \text { légèrement côtelée }\end{array} & \text { Jaune-orange } \\ 12 & \text { EWSGO } & \text { Padma F1 } & \text { TM44/14EW } & \text { Cordiforme } & \text { Orange } \\ 13 & \text { AVRDC } & \text { FMTT 847 } & \text { TM16/04A } & \text { Ellipsoïde } & \text { Jaune-orange } \\ 14 & \text { AVRDC } & \text { FMTT 848 } & \text { TM17/04A } & \text { Ronde } & \text { Jaune-orange } \\ 15 & \text { AVRDC } & \text { CLIN 2418A } & \text { TM48/04A } & \text { Ellipsoïde } & \text { Rouge }\end{array}$

RESULTATS

Analyse de variance des paramètres agromorphologiques de 15 lignées de tomate

\section{Développement végétatif des plants}

Le Tableau 2 illustre les taux de reprise des plants au champ 15 jours après le repiquage, l'état végétatif des plants 30 jours après le repiquage et la hauteur des plants au stade de la floraison. L'analyse de variance a montré qu'il n'y a pas de différences significatives entre les lignées en ce qui concerne les pourcentages de reprise des plants après repiquage. Par contre, une variabilité a été notée entre les lignées pour l'état végétatif et la hauteur des plants. Les plants, les plus vigoureux ont été ceux des lignées TM41/14EW, PETOM, TM17/04A, TM44/14EW, TM16/04A et TM43/14EW. Les plants des lignées TM47/15BF et TM48/04A ont eu un développement végétatif qualifié de mauvais. Pour la hauteur des plants, la taille des plants au stade de la floraison a varié entre 41 et $62 \mathrm{~cm}$. La plus petite taille des plants a été obtenue avec la lignée TMA97 et la plus grande taille a été produite par la lignée TM41/14EW.

\section{Phénologie des différentes lignées de tomate}

Le Tableau 3 regroupe le délai de floraison et la date de la première récolte de 15 lignées de tomate. Les résultats obtenus ont montré qu'il n'existe aucune différence significative entre les lignées en ce qui concerne le délai de première floraison. Par contre, pour le délai de floraison, une variabilité entre les lignées a été observée. Ce délai a varié entre 52 et 64 JAS. Les lignées TM43/14EW, TM1/04A, TM16/04A,
TM44/14EW, TMA97, PETOM et LINDO ont fleuri entre 52 et $56 \mathrm{JAS}$, alors que les lignées TM40/14EW, TM42/14EW, TM45/15BF, TM46/15BF, TM41/EW, TM39/14EW, TM47/15BF et TM48/04A ont fleuri entre 59 et 64 JAS.

\section{Composantes du rendement et fermeté des} fruits

Dans le Tableau 4 sont consignés le nombre total de fruits récoltés puis les taux de fruits avariés et de fruits sains. L'analyse de variance a montré une variabilité entre les lignées pour tous les composantes du rendement. Le nombre total de fruits récoltés a varié entre 110 et 534 fruits. La lignée TM44/14EW a produit le plus grand nombre avec 534 fruits récoltés et la lignée TM48/04A en a donné le plus petit nombre avec 110. Concernant le poids total de fruits, la plus importante valeur $(23567 \mathrm{~g})$ a été enregistrée chez la lignée TM40/14EW; tandis que le plus petit poids de fruits (6167) a été fourni par TM48/04A. Quant aux taux de fruits avariés, la lignée LINDO, a enregistré le taux le plus élevé $(59,28 \%)$. Cependant, la lignée TM46/15BF a obtenu le taux de fruits avariés le plus faible $(13,39 \%)$. L'analyse du taux de fruits sains a montré que les valeurs ont varié entre 40 et $86,61 \%$. Les taux le plus élevés ont été notés chez TM46/15BF et TM45/15B puis les plus faibles ont été donnés par TM16/04A et LINDO.

Le Tableau 5 présente le nombre de fruits par plant, le poids moyen d'un fruit, les rendements potentiel et net puis la fermeté des fruits des 15 lignées de tomate. L'analyse de variance a montré une différence hautement 
significative pour ces paramètres évalués. Le plus petit nombre de fruits/plant (5) a été enregistré chez les ignées TM39/14EW et TM48/04A ; alors que la lignée TM40/14EW en a produit le plus important nombre (25 fruits /plant). Le poids moyen du fruit le plus élevé $(60,53 \mathrm{~g})$ a été généré par TM39/14EW ; alors que les plus faibles poids moyens $(32,32$ à $36,99 \mathrm{~g}$ ) ont été produits par LINDO, TMA97, TM45/15BF, TM46/15BF, TM47/15BF et TM40/14EW.

En ce qui concerne le rendement potentiel, l'analyse de variance a montré que la lignée TM44/14EW a été la plus performante avec 47,13 t/ha. Les lignées TMA97, TM46/15BF, PETOM, LINDO, TM41/14EW, TM39/14EW et TM48/04A ont eu les rendements potentiels les plus faibles compris entre 21,33 et $12,33 \mathrm{t} / \mathrm{ha}$.

Quant au rendement net, l'analyse des données a indiqué que les lignées TM44/14EW, TM40/14EW, TM43/4EW et TM42/14EW introduites d'East West Seed Global Orion ont été les plus productives avec un rendement moyen de plus de 30 t/ha. Les lignées TM39/14EW, LINDO et TM48/04A ont été moins productives avec moins de 10 t/ha.

Pour la fermeté des fruits, trois niveaux ont été distingués. Les lignées TM43/14EW et TM44/14EW ont obtenu des fruits très fermes avec une note de 5/5. TM16/04A, TM40/14EW, TM424/14EW, TM45/15BF, TM46/15BF, TM47/15BF, TMA97, LINDO et PETOM ont présenté des fruits fermes avec une note 4/5. TM17/04A, TM39/14EW, TM41/14EW et TM48/04A ont donné des fruits moyennement fermes avec une note $3 / 5$.

Corrélations entre les paramètres
agromorphologiques des 15 lignées de
tomate

Une matrice de corrélation de Pearson a été générée pour déterminer le niveau de relation entre les variables agromorphologiques étudiées. Seules les corrélations significatives $(P<0,05)$ ont été mises en relief dans le Tableau 6. L'analyse de ce tableau a montré une corrélation forte et positive entre le développement végétatif (Et veg) et les composantes du rendement (nombre et poids total de fruits par plant), les rendements puis la fermeté des fruits. Pour les stades phénologiques, c'est le délai de floraison (D F) qui a été plus corrélé de façon positive avec le rendement et ses composantes $(0,88$ à 0,98). Dans cette matrice, les corrélations négatives $(r=-1)$ ont été établies entre le taux de fruits sains et le taux de fruits avariés. Aussi, le poids moyen du fruit a été négativement lié $(r=-0,52)$ au nombre total de fruits et au nombre de fruits par plant.

Analyse de la diversité de 15 lignées de tomate par la classification hiérarchique ascendante (CAH)

Pour analyser la structuration de la variabilité au sein de la collection de tomate, une classification hiérarchique ascendante a été effectuée selon la méthode de Ward (1963). Le dendrogramme réalisé à partir des moyennes de quatorze caractères quantitatifs mesurés a permis d'identifier trois groupes de diversité agromorphologique (Figure 5). Il s'agit du groupe I (TMA97, TM47/15BF et TM48/04A) caractérisé par un délai de floraison précoce, du groupe II (TM39/14EW, TM40/14EW, TM41/14EW, TM42/14EW, TM45/15BF, TM46/15BF, LINDO et PETOM) qui a obtenu une performance moyenne des paramètres agromorphologiques mesurés et du groupe III (TM16/04A, TM17/04A, TM43/14EW et TM44/14EW) dont le développement végétatif a été le plus vigoureux avec des valeurs du rendement et ses composantes qui ont été les plus élevées. Ce groupe renferme également les lignées dont les fruits ont été les plus fermes. Sur la base des valeurs élevées du coefficient de Fisher $(F>10)$, les variables état végétatif des plants (Et veg), délai de floraison (DF), nombre total de fruits (Nb Tft), poids total des fruits (Pds Tft), (nombre de fruits par plant $(\mathrm{Nb} \mathrm{ft} / \mathrm{p})$, rendement potentiel (Rdt pot), rendement net (Rdt net) et fermeté des fruits (Ferm), ont été les plus discriminantes (Tableau 7). 
Tableau 2: Taux de reprise, état végétatif et hauteur des plants des 15 lignées de tomate.

\begin{tabular}{|c|c|c|c|}
\hline Lignées & $\begin{array}{l}\text { Taux de reprise au } \\
\text { repiquage }(\%)\end{array}$ & $\begin{array}{l}\text { Etats végétatif à } \\
30 \text { JAR (1 à } 5)\end{array}$ & $\begin{array}{l}\text { Hauteurs des plants à } \\
\text { la floraison }(\mathbf{c m})\end{array}$ \\
\hline TM47/15BF & $93,93 \pm 3,03^{\mathrm{a}^{*}}$ & $2,6 \pm 0,33 a^{*}$ & $54 \pm 3,48^{\mathbf{b c}^{*}}$ \\
\hline TM48/04A & $90,90 \pm 2,62^{\mathrm{a}}$ & $2,6 \pm 0,33^{\mathrm{a}}$ & $52 \pm 1,45^{\mathrm{bc}}$ \\
\hline TMA97 & $100 \pm 0,00^{\mathbf{a}}$ & $3 \pm 0,00^{\mathbf{a b}}$ & $41 \pm 4,58^{\mathbf{a b}}$ \\
\hline TM46/15BF & $95,45 \pm 2,62^{\mathrm{a}}$ & $3 \pm 0,00^{\mathbf{a b}}$ & $56 \pm 4,93^{b c}$ \\
\hline LINDO & $100 \pm 0,00^{\mathbf{a}}$ & $3 \pm 0,00^{\mathbf{a b}}$ & $60 \pm 4,84^{\mathrm{bc}}$ \\
\hline TM39/14EW & $92,42 \pm 4,01^{\mathrm{a}}$ & $3 \pm 0,00^{\mathbf{a b}}$ & $49 \pm 16,34^{\text {abc }}$ \\
\hline TM40/14EW & $96,96 \pm 3,03^{\mathbf{a}}$ & $3,3 \pm 0,33^{\mathbf{a b c}}$ & $58 \pm 6,01^{\text {bc }}$ \\
\hline TM45/15BF & $98,48 \pm 1,51^{\mathrm{a}}$ & $3,3 \pm 0,33^{\mathrm{abc}}$ & $58 \pm 5,04^{\mathrm{bc}}$ \\
\hline TM42/14EW & $96,96 \pm 3,03^{a}$ & $3,3 \pm 0,33^{\text {abc }}$ & $42 \pm 5,29^{\mathrm{abc}}$ \\
\hline TM41/14EW & $100 \pm 0,00^{\mathbf{a}}$ & $3,6 \pm 0,33^{\text {bcd }}$ & $62 \pm 4,40^{\mathrm{c}}$ \\
\hline PETOM & $93,93 \pm 6,06^{\mathbf{a}}$ & $3,6 \pm 0,33^{\text {bcd }}$ & $49 \pm 2,08^{\mathbf{a b c}}$ \\
\hline TM17/04A & $100 \pm 0,00^{\mathbf{a}}$ & $3,6 \pm 0,33^{\mathrm{bcd}}$ & $43 \pm 4,40^{\mathbf{a b c}}$ \\
\hline TM44/14EW & $100 \pm 0,00^{\mathbf{a}}$ & $4 \pm 0,00^{\text {cde }}$ & $58 \pm 1,45^{\text {bc }}$ \\
\hline TM16/04A & $100 \pm 0,00^{\mathbf{a}}$ & $4,3 \pm 0,33^{\mathrm{de}}$ & $55 \pm 2,40^{\mathbf{b c}}$ \\
\hline TM43/14EW & $100 \pm 0,00^{\mathbf{a}}$ & $4,6 \pm 0,33^{\mathrm{e}}$ & $58 \pm 4,40^{\mathbf{b c}}$ \\
\hline Moyenne & $97,27 \pm 0,71$ & $3,4 \pm 0,10$ & $52 \pm 1,75$ \\
\hline Probabilité & 0,13 & 0,00000 & 0,04 \\
\hline $\mathrm{C} \mathrm{V}(\%)$ & 5,69 & 29,73 & 28,36 \\
\hline
\end{tabular}

*Les moyennes du taux de reprise des plants après repiquage, de l'état végétatif et de la hauteur des plants en colonne affectées de la même lettre ne diffèrent pas significativement au seuil de 5\% (test de Duncan).

Tableau 3: Délai de floraison et date de la 1ère récolte de 15 lignées de tomate.

\begin{tabular}{ccc}
\hline Variétés & Délai de floraison en JAS & Délai 1ère récolte en JAS \\
\hline TM43/14EW & $52 \pm 1^{\mathbf{a}}$ & $94 \pm 0^{\mathbf{a}}$ \\
TM17/04A & $53 \pm 2^{\mathbf{a}}$ & $94 \pm 0^{\mathbf{a}}$ \\
TM16/04A & $55 \pm 1^{\mathbf{a}}$ & $94 \pm 0^{\mathbf{a}}$ \\
TM44/14EW & $55 \pm 1^{\mathbf{a}}$ & $94 \pm 0^{\mathbf{a}}$ \\
TMA97 & $56 \pm 2^{\mathbf{a}}$ & $94 \pm 0^{\mathbf{a}}$ \\
PETOM & $56 \pm 1^{\mathbf{a}}$ & $94 \pm 0^{\mathbf{a}}$ \\
LINDO & $56 \pm 1^{\mathbf{a}}$ & $94 \pm 0^{\mathbf{a}}$ \\
TM40/14EW & $59 \pm 3^{\mathbf{a b}}$ & $97 \pm 1^{\mathbf{a}}$ \\
TM42/14EW & $59 \pm 2^{\mathbf{a b}}$ & $97 \pm 0^{\mathbf{a}}$ \\
TM45/15BF & $59 \pm 4^{\mathbf{a b}}$ & $94 \pm 0^{\mathbf{a}}$
\end{tabular}




\begin{tabular}{ccc} 
TM46/15BF & $61 \pm 4^{\mathbf{b}}$ & $94 \pm 0^{\mathbf{a}}$ \\
TM41/14EW & $61 \pm 1^{\mathbf{b}}$ & $95 \pm 1^{\mathbf{a}}$ \\
TM39/14EW & $63 \pm 2^{\mathbf{b}}$ & $98 \pm 0^{\mathbf{a}}$ \\
TM47/15BF & $63 \pm 2^{\mathbf{b}}$ & $101 \pm 7^{\mathbf{a}}$ \\
TM48/04A & $64 \pm 1^{\mathbf{b}}$ & $94 \pm 0^{\mathbf{a}}$ \\
\hline Moyenne & $58 \pm 2$ & $95 \pm 2,98$ \\
Probabilité & 0,005 & 0,200 \\
C V $(\%)$ & 30,23 & 29,44
\end{tabular}

*Les moyennes des valeurs des stades phénologiques en colonne affectées de la même lettre ne diffèrent pas significativement au seuil de 5\% (test de Duncan).

Tableau 4: Nombre total de fruits récoltés, taux de fruits avariés et sains des 15 lignées de tomate.

\begin{tabular}{|c|c|c|c|c|}
\hline Variétés & $\begin{array}{l}\text { Nombre total de } \\
\text { fruits récoltés }\end{array}$ & $\begin{array}{l}\text { Poids total des } \\
\text { fruits (g) }\end{array}$ & $\begin{array}{l}\text { Taux de fruits } \\
\text { avariés (\%) }\end{array}$ & $\begin{array}{c}\text { Taux de fruits } \\
\text { sains }(\%)\end{array}$ \\
\hline TM48/04A & $110 \pm 7^{7^{*}}$ & $6166,67 \pm 420,64^{\mathrm{a}^{*}}$ & $28,29 \pm 0,35^{\text {abc* }^{*}}$ & $71,71 \pm 0,35^{\text {abc* }}$ \\
\hline TM39/14EW & $114 \pm 38^{a}$ & $6900 \pm 2300^{a}$ & $42,10 \pm 14,03^{\mathrm{cd}}$ & $57,90 \pm 14,03^{\mathrm{ab}}$ \\
\hline TM41/14EW & $168 \pm 56^{\mathrm{ab}}$ & $8266,67 \pm 3229,72^{a}$ & $28,98 \pm 4,10^{\mathbf{a b c}}$ & $71,02 \pm 4,10^{\mathbf{a b c}}$ \\
\hline TM17/04A & $202 \pm 16^{\mathbf{a b}}$ & $9633,33 \pm 1452,96^{\mathbf{a}}$ & $32,42 \pm 9,63^{\mathrm{abc}}$ & $67,58 \pm 9,63^{\mathrm{abc}}$ \\
\hline TM16/04A & $234 \pm 48^{\text {abc }}$ & $10116,67 \pm 3265,51^{\mathrm{a}}$ & $54,29 \pm 5,25^{\mathrm{d}}$ & $45,71 \pm 5,25^{\mathrm{a}}$ \\
\hline PETOM & $242 \pm 54^{\text {abc }}$ & $10483,33 \pm 1369,41^{\mathbf{a}}$ & $29,62 \pm 6,76^{\mathrm{abc}}$ & $70,38 \pm 6,76^{\mathrm{abc}}$ \\
\hline LINDO & $270 \pm 50^{\mathbf{a b c}}$ & $10666,67 \pm 2298,06^{\mathbf{a}}$ & $59,28 \pm 10,97^{\mathbf{d}}$ & $40,72 \pm 10,97^{\mathbf{a}}$ \\
\hline TMA97 & $293 \pm 60^{\text {abc }}$ & $11083,33 \pm 2834,36^{\mathbf{a b}}$ & $35,07 \pm 4,97^{\mathbf{b c}}$ & $64,93 \pm 4,97^{\mathbf{b c}}$ \\
\hline TM46/15BF & $329 \pm 55^{\mathrm{abc}}$ & $11416,67 \pm 1211,17^{\mathrm{ab}}$ & $13,39 \pm 2,21^{\mathbf{a}}$ & $86,61 \pm 2,21^{\mathrm{c}}$ \\
\hline TM45/15BF & $338 \pm 85^{\text {abc }}$ & $12963,33 \pm 3162,98^{\mathbf{a b}}$ & $14,25 \pm 2,50^{\mathbf{a}}$ & $85,75 \pm 2,50^{\mathbf{c}}$ \\
\hline TM42/14EW & $350 \pm 76^{\text {abc }}$ & $13966,67 \pm 9081,36^{\mathbf{a b}}$ & $23,74 \pm 1,23^{\text {abc }}$ & $76,26 \pm 1,23^{\mathrm{abc}}$ \\
\hline TM47/15BF & $373 \pm 235^{\text {abc }}$ & $18116,67 \pm 4818,56^{\mathbf{a b}}$ & $18,63 \pm 2,52^{\mathrm{ab}}$ & $81,37 \pm 2,52^{\mathbf{b c}}$ \\
\hline TM43/14EW & $474 \pm 40^{\text {bc }}$ & $18266,67 \pm 1856,61^{\mathrm{ab}}$ & $20,39 \pm 2,81^{\mathbf{a b}}$ & $79,61 \pm 2,81^{\text {bc }}$ \\
\hline TM44/14EW & $477 \pm 93^{\text {bc }}$ & $19083,33 \pm 6066,46^{\mathbf{a b}}$ & $32,14 \pm 11,88^{\mathbf{a b c}}$ & $67,85 \pm 11,88^{\text {abc }}$ \\
\hline TM40/14EW & $534 \pm 189^{c}$ & $23566,67 \pm 5212,51^{\mathbf{b}}$ & $23,45 \pm 1,71^{\mathbf{a b c}}$ & $76,55 \pm 1,71^{\mathrm{abc}}$ \\
\hline Moyenne & $301 \pm 28$ & $12711,11 \pm 1150,90$ & $30,41 \pm 2,44$ & $69,59 \pm 2,44$ \\
\hline Probabilité & 0,04 & 0,02 & 0,00000 & 0,00000 \\
\hline $\mathrm{C} \mathrm{V}(\%)$ & 29,52 & 267,76 & 25,49 & 32,99 \\
\hline
\end{tabular}

*Les moyennes des composantes du rendement en colonne affectées de la même lettre ne diffèrent pas significativement au seuil de 5\% (test de Duncan). 
Tableau 5: Nombre de fruits par plant, poids moyen du fruit, rendements potentiel et net puis fermeté des fruits des 15 lignées de tomate.

\begin{tabular}{|c|c|c|c|c|c|}
\hline Variétés & $\begin{array}{l}\text { Nombre de } \\
\text { fruits par plant }\end{array}$ & $\begin{array}{l}\text { Poids moyen } \\
\text { du fruit (g) }\end{array}$ & $\begin{array}{c}\text { Rendement } \\
\text { potentiel (t/ha) }\end{array}$ & $\begin{array}{l}\text { Rendement } \\
\text { net (t/ha) }\end{array}$ & Fermeté \\
\hline TM39/14EW & $5 \pm 2^{\mathrm{a}^{*}}$ & $60,53 \pm 5,34^{\mathrm{d}^{*}}$ & $13,8 \pm 4,60^{\mathbf{a}^{*}}$ & $6,8 \pm 2,26^{\mathbf{a}^{*}}$ & $3 \pm 0,00^{\mathbf{a}}$ \\
\hline TM48/04A & $5 \pm 1^{\mathrm{a}}$ & $56,25 \pm 0,14^{\mathrm{cd}}$ & $12,33 \pm 0,84^{\mathbf{a}}$ & $9,93 \pm 0,74^{\mathrm{a}}$ & $3 \pm 0,00^{\mathrm{a}}$ \\
\hline TM41/14EW & $7 \pm 3^{\mathrm{a}}$ & $49,02 \pm 5,13^{\mathbf{b c}}$ & $16,53 \pm 6,45^{\mathrm{a}}$ & $13,33 \pm 5,66^{\mathbf{a b}}$ & $3 \pm 0,00^{\mathrm{a}}$ \\
\hline TM17/04A & $9 \pm 1^{a b}$ & $56,23 \pm 1,66^{\mathrm{cd}}$ & $22,83 \pm 2,42^{\mathbf{a b}}$ & $16,50 \pm 1,66^{\mathbf{a b}}$ & $3 \pm 0,00^{\mathrm{a}}$ \\
\hline TM16/04A & $11 \pm 2^{\mathrm{abc}}$ & $54,35 \pm 6,16^{\mathrm{cd}}$ & $25,86 \pm 6,32^{\mathbf{a b}}$ & $16,70 \pm 5,08^{\mathbf{a b}}$ & $\begin{array}{c}3,66 \pm 1,66 \\
\text { b }\end{array}$ \\
\hline PETOM & $12 \pm 2^{\mathrm{abc}}$ & $39,55 \pm 5,15^{\mathbf{a b}}$ & $20,23 \pm 6,53^{\mathrm{a}}$ & $16,40 \pm 5,51^{\mathbf{a b}}$ & $4 \pm 0,00 b^{c}$ \\
\hline LINDO & $12 \pm 2^{\mathrm{abc}}$ & $36,22 \pm 1,83^{\mathrm{a}}$ & $19,26 \pm 2,90^{\mathbf{a}}$ & $9,86 \pm 1,33^{\mathbf{a}}$ & $4 \pm 0,00^{\mathbf{b c}}$ \\
\hline TMA97 & $13 \pm 3^{\mathrm{abc}}$ & $36,19 \pm 0,42^{\mathrm{a}}$ & $21,33 \pm 4,59^{\mathbf{a}}$ & $15,46 \pm 3,20^{\mathbf{a b}}$ & $4 \pm 0,00^{\mathbf{b c}}$ \\
\hline TM45/15BF & $15 \pm 4^{\mathrm{abc}}$ & $32,57 \pm 2,05^{\mathrm{a}}$ & $22,16 \pm 5,66^{\mathbf{a b}}$ & $20,08 \pm 5,34^{\mathbf{a b}}$ & $4 \pm 0,00^{\mathbf{b c}}$ \\
\hline TM46/15BF & $16 \pm 3^{\mathrm{abc}}$ & $32,32 \pm 1,95^{\mathrm{a}}$ & $20,96 \pm 2,73^{\mathrm{a}}$ & $18,96 \pm 2,32^{\mathbf{a b}}$ & $4 \pm 0,00^{\mathbf{b c}}$ \\
\hline TM42/14EW & $16 \pm 3^{\mathrm{abc}}$ & $59,39 \pm 7,16^{\mathrm{cd}}$ & $36,23 \pm 9,63^{\mathbf{a b}}$ & $31,80 \pm 8,79^{\mathbf{b}}$ & $4 \pm 0,00^{\mathbf{b c}}$ \\
\hline TM47/15BF & $17 \pm 11^{\text {abc }}$ & $35,39 \pm 1,99^{\mathbf{a}}$ & $27,93 \pm 18,66^{\mathbf{a b}}$ & $23,56 \pm 15,18^{\mathbf{a b}}$ & $4 \pm 0,00^{\mathbf{b c}}$ \\
\hline TM44/14EW & $22 \pm 4^{\text {bc }}$ & $49,08 \pm 1,62^{\mathbf{b c}}$ & $47,13 \pm 10,22^{\mathbf{b}}$ & $34,26 \pm 6,83^{\mathbf{b}}$ & $4,33 \pm 0,33^{\mathrm{c}}$ \\
\hline TM43/14EW & $22 \pm 2^{\mathbf{b c}}$ & $38,66 \pm 3,14^{\mathrm{ab}}$ & $36,53 \pm 3,71^{\mathbf{a b}}$ & $31,83 \pm 3,69^{\mathbf{b}}$ & $5 \pm 0,00^{d}$ \\
\hline TM40/14EW & $25 \pm 8^{c}$ & $36,99 \pm 1,82^{\mathrm{a}}$ & $38,16 \pm 12,13^{\mathbf{a b}}$ & $33,13 \pm 10,42^{\mathbf{b}}$ & $5 \pm 0,00^{d}$ \\
\hline Moyenne & $14 \pm 2$ & $44,32 \pm 1,60$ & $25,42 \pm 2,30$ & $19,91 \pm 1,95$ & $3,86 \pm 0,15$ \\
\hline Probabilité & 0,004 & 0,00000 & 0,017 & 0,004 & 0,00000 \\
\hline $\mathrm{C} \mathrm{V}(\%)$ & 23,64 & 38,31 & 27,76 & 28,79 & 29,26 \\
\hline
\end{tabular}

*Les moyennes des valeurs du rendement et ses composantes en colonne affectées de la même lettre ne diffèrent pas significativement au seuil de 5\% (test de Duncan). 
N. D. COULIBALY et al. /Int. J. Biol. Chem. Sci. 13(3): 1565-1581, 2019

Tableau 6: Matrice de corrélation de Pearson (r) entre les paramètres agromorphologiques analysés chez 15 lignées de tomate.

\begin{tabular}{|c|c|c|c|c|c|c|c|c|c|c|c|c|c|c|}
\hline & Tx rep & Et veg & $\mathrm{HP}$ & DF & D 1re & $\mathrm{N} \mathrm{Tft}$ & P Tft & $\mathrm{T}$ fa & $\mathrm{T}$ fst & $\mathrm{N} \mathrm{f/p}$ & $\mathrm{Pm} \mathrm{ft}$ & R pot & R net & Ferm \\
\hline Tx rep & 1.00 & & & & & & & & & & & & & \\
\hline Et veg & $0.62 *$ & 1.00 & & & & & & & & & & & & \\
\hline HP & 0.18 & 0.22 & 1.00 & & & & & & & & & & & \\
\hline DF & $0.59 *$ & $0.95^{*}$ & 0.10 & 1.00 & & & & & & & & & & \\
\hline $\mathrm{D} 1^{\mathrm{re}}$ & 0.37 & 0.50 & 0.03 & $0.55^{*}$ & 1.00 & & & & & & & & & \\
\hline $\mathrm{Nb} \mathrm{Tft}$ & 0.60 & $0.98 *$ & 0.19 & $0.98 *$ & $0.54^{*}$ & 1.00 & & & & & & & & \\
\hline P Tft & $0.58 *$ & $0.96 *$ & 0.14 & $0.94 *$ & 0.43 & $0.96 *$ & 1.00 & & & & & & & \\
\hline $\mathrm{T}$ fa & -0.17 & -0.36 & 0.36 & -0.46 & -0.20 & -0.40 & -0.38 & 1.0 & & & & & & \\
\hline $\mathrm{T}$ fst & 0.17 & 0.36 & -0.36 & 0.46 & 0.20 & 0.40 & 0.38 & $-1.0 *$ & 1.0 & & & & & \\
\hline $\mathrm{N} \mathrm{f/p}$ & $0.58 *$ & $0.97 *$ & 0.21 & $0.97 *$ & $0.52 *$ & $1.00 *$ & $0.96^{*}$ & -0.4 & 0.4 & 1.00 & & & & \\
\hline $\mathrm{Pm} \mathrm{ft}$ & -0.48 & -0.48 & 0.02 & -0.48 & -0.32 & $-0.52 *$ & -0.45 & 0.3 & -0.3 & $-0.52 *$ & 1.00 & & & \\
\hline R pot & 0.47 & $0.84 *$ & 0.21 & $0.88 *$ & 0.49 & $0.88 *$ & $0.85^{*}$ & -0.3 & 0.3 & $0.87 *$ & -0.09 & 1.00 & & \\
\hline $\mathrm{R}$ net & 0.44 & $0.88^{*}$ & 0.06 & $0.93 *$ & 0.49 & $0.91 *$ & $0.89 *$ & -0.5 & 0.5 & $0.91 *$ & -0.18 & $0.95 *$ & 1.00 & \\
\hline Ferm & 0.49 & $0.91 *$ & 0.25 & $0.89 *$ & $0.56^{*}$ & $0.93 *$ & $0.88 *$ & -0.2 & 0.2 & $0.94 *$ & $-0.62 *$ & $0.74 *$ & 0.77 & 1.00 \\
\hline
\end{tabular}

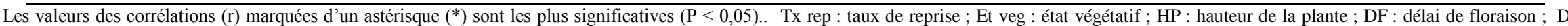

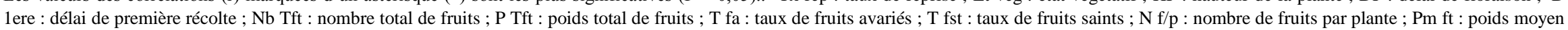
du fruit ; R pot : rendement potentiel ; R net : rendement net ; Ferm : fermeté des fruits. 


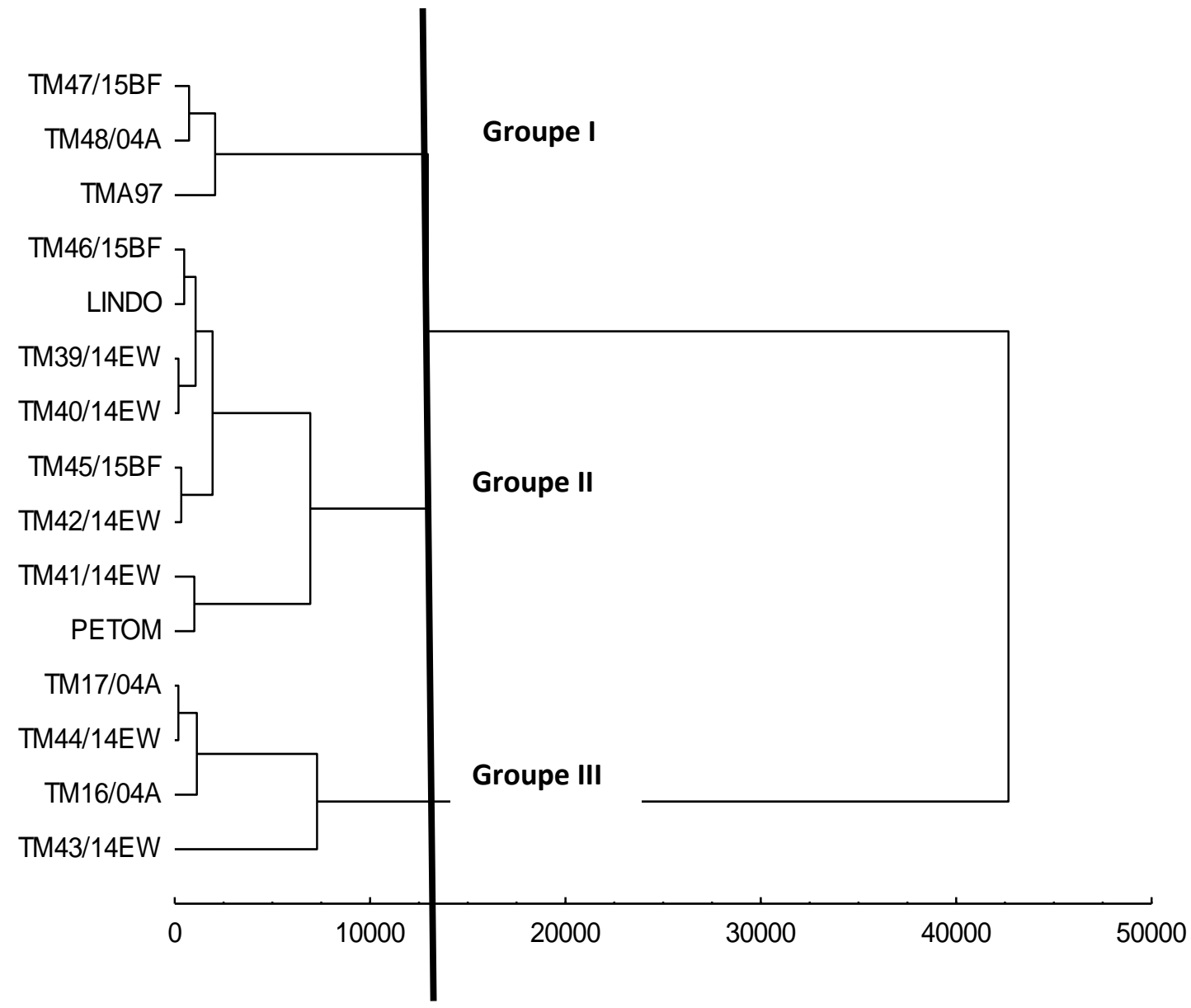

Figure 5: Dendrogramme issu de la Classification Hiérarchique Ascendante (CHA) de 15 lignées de tomate selon la méthode de Ward (1963).

Tableau 7 : Caractéristiques principales des différents groupes de lignées de tomate formés par la Classification Hiérarchique Ascendante (CHA).

\begin{tabular}{llllll}
\hline \multirow{2}{*}{ Paramètres } & \multicolumn{1}{c}{ Groupes } & \multicolumn{1}{c}{ Statistiques } \\
\cline { 2 - 6 } & \multicolumn{1}{c}{ I } & \multicolumn{1}{c}{ II } & \multicolumn{1}{c}{ III } & \multicolumn{1}{c}{$\boldsymbol{F}$} \\
\hline Tx rep & $94,33 \pm 4,63^{\mathrm{a}}$ & $96,77 \pm 2,74^{\mathrm{a}}$ & $100 \pm 006^{\mathrm{a}}$ & 3,01527 & 0,08690 \\
Et veg & $2,73 \pm 0,23^{\mathrm{a}}$ & $3,26 \pm 0,25^{\mathrm{a}}$ & $4,12 \pm 0,42^{\mathrm{c}}$ & 19,67808 & $0,00016^{* * *}$ \\
HP & $49,00 \pm 7,00^{\mathrm{a}}$ & $54,25 \pm 6,86^{\mathrm{a}}$ & $53,50 \pm 7,149^{\mathrm{a}}$ & 0,63566 & 0,54652 \\
DF & $53,33 \pm 1,52^{\mathrm{a}}$ & $57,62 \pm 2,136^{\mathrm{ab}}$ & $62,75 \pm 1,25^{\mathrm{b}}$ & 22,73219 & $0,00008^{* * *}$ \\
D 1ere rec & $94,00 \pm 0,00^{\mathrm{a}}$ & $94,75 \pm 1,38^{\mathrm{a}}$ & $97,00 \pm 3,16^{\mathrm{a}}$ & 2,60690 & 0,11477 \\
Nb Tft & $130,66 \pm 32,390^{\mathrm{a}}$ & $282,25 \pm 54,16^{\mathrm{b}}$ & $464,50 \pm 66,95^{\mathrm{c}}$ & 32,71902 & $0,00001_{* *}$ \\
Pds Tft & $7111 \pm 1065^{\mathrm{a}}$ & $11291 \pm 1472^{\mathrm{b}}$ & $19758 \pm 2574^{\mathrm{c}}$ & 49,63637 & $0,00000^{* * *}$ \\
Tx ft av & $33,12 \pm 7,78^{\mathrm{a}}$ & $32,75 \pm 16,82^{\mathrm{a}}$ & $23,65 \pm 5,99^{\mathrm{a}}$ & 0,67543 & 0,52727 \\
Tx ft st & $66,87 \pm 7,78^{\mathrm{a}}$ & $67,24 \pm 16,82^{\mathrm{a}}$ & $76,34 \pm 6,00^{\mathrm{a}}$ & 0.67501 & 0,52747
\end{tabular}




\begin{tabular}{llllll} 
Nb ft/p & $5,66 \pm 1,15^{\mathrm{a}}$ & $13,00 \pm 2,50^{\mathrm{b}}$ & $21,50 \pm 3,31^{\mathrm{c}}$ & 33,19331 & $0,00001^{* * *}$ \\
Pds moy ft & $55,26 \pm 5,81^{\mathrm{a}}$ & $43,35 \pm 11,33^{\mathrm{a}}$ & $40,03 \pm 6,17^{\mathrm{a}}$ & 2,42253 & 0,13069 \\
Rdt pot & $14,22 \pm 2,13^{\mathrm{a}}$ & $23,60 \pm 5,47^{\mathrm{b}}$ & $37,43 \pm 7,86^{\mathrm{c}}$ & 14,54523 & $0,00062^{* * *}$ \\
Rdt net & $10,02 \pm 3,26^{\mathrm{a}}$ & $18,22 \pm 6,26^{\mathrm{b}}$ & $30,69 \pm 4,85^{\mathrm{c}}$ & 12,78855 & $0,00106^{* *}$ \\
Ferm & $3,00 \pm 0,00 \mathrm{a}$ & $3,83 \pm 0,35^{\mathrm{b}}$ & $4,56 \pm 0,50^{\mathrm{c}}$ & 15,74971 & $0,00044 * * *$ \\
\hline
\end{tabular}

$\mathrm{P}<0,001$ : Probabilité très hautement significative***; $\mathrm{P}<0,01$ : Probabilité hautement significative** $; \mathrm{P}<0,05$ : Probabilité significative*; Tx rep : taux de reprise des plants après repiquage ; Et veg : état végétatif des plants 30 jours après repiquage ; HP : hauteur des plants; $\mathrm{DF}$ : délai de floraison; D 1ere rec : délai de première récolte ; $\mathrm{Nb} \mathrm{Tft}:$ nombre total de fruits ; Pds Tft : poids total de fruits ; Tx ft av : taux de fruit avariés ; Tx ft st : taux de fruits saints ; Nb ft/p : nombre de fruits par plante ; Pds moy ft : poids moyen d'un fruit; Rdt pot : rendement potentiel ; Rdt net : rendement net ; Ferm : fermeté des fruits.

Dans une même colonne, les moyennes suivis d'une même lettre ne sont pas statistiquement identiques au seuil de 5\% (test de Fisher).

\section{DISCUSSION}

\section{Stades végétatifs et phénologiques des différentes lignées de tomate}

Le taux de reprise des plantules au champ a été élevé sur l'ensemble des parcelles. La sélection des plantules vigoureuses opérée avant le repiquage justifierait cette bonne reprise. En effet, il est probable que la vigueur des plantules en pépinière ait influencé leur reprise en plantation. De nombreuses études, notamment celles de Muhammad et al. (2007), Ojetayo et al. (2010) puis Musas (2012) ont montré que les taux de reprise du chou pommé, de la tomate, de l'épinard et de l'oignon étaient similaires sur des sols fertilisés et non fertilisés. Ils ont montré qu'en effet la reprise des plantules ne dépend pas des éléments minéraux contenus dans le sol. Les résultats ont indiqué que les lignées TM41/14EW, PETOM, TM17/04A, TM44/14EW, TM16/04A et TM43/14EW ont eu un très bon développement végétatif. Par contre, les lignées TM47/15BF et TM48/04A ont présenté un mauvais développement végétatif. Au niveau de la hauteur des plants, les résultats ont montré que la taille des plants a varié entre 41 et $62 \mathrm{~cm}$. La lignée locale TMA97 a donné la plus petite taille, tandis TM41/14EW, une lignée introduite a eu la plus importante taille. Ces résultats indiquent que la croissance en hauteur des plantes de tomate ne semble pas être influencée par l'origine des lignées, en témoigne la faible hauteur de TMA97, la lignée locale et la grande taille des plantes de TM41/14EW originaire d'un autre pays (Norvège). La hauteur des plantes dépendrait plutôt des caractères intrinsèques de chaque lignée et surtout des conditions pédoclimatiques particulières leur permettant de développer au maximum leurs potentialités de croissance. La variabilité observée par rapport au développement végétatif et la hauteur des plantes résulterait donc de la différence dans leur capacité d'adaptation au milieu. Les travaux de Fondio et al. (2013) sur l'évaluation des performances agronomiques de neuf variétés de tomate ont montré que la différence observée dans la croissance de diverses variétés de tomate serait liée à leur génotype et à l'environnement dans lequel elles ont été testées.

Concernant les stades phénologiques, les résultats ont distingué des lignées précoces et tardives en rapport avec les délais de floraison et de la première récolte. $\mathrm{La}$ variabilité qui a été observée au niveau des stades phénologiques chez les lignées, pourrait être liée à l'interaction des différents génotypes et l'environnement. En effet, de la floraison jusqu'au murissement des fruits, plusieurs phénomènes physiologiques et biochimiques interviennent parmi lesquels, il y a la mobilisation des éléments minéraux pour l'initiation florale et le remplissage des fruits. De ce fait, la capacité de chaque lignée à puiser les éléments minéraux et l'eau puis à les transformer pour les mettre à la 
disposition des organes de la plante sous l'effet des facteurs environnementaux (lumière, température...) pourrait fortement influencer la durée de floraison, la fructification et le murissement des fruits. Les travaux de Gwennaël (2013) ont également rapporté que le phénotype qui est l'expression visible du génotype peut être influencé par des facteurs externes. En effet, selon lui, l'expression d'un caractère donné peut être le résultat de l'interaction entre les facteurs génétiques et ceux du milieu de vie de la plante.

\section{Rendements, composantes du rendement et fermeté des fruits}

Les résultats obtenus en évaluant les rendements et leurs composantes ont montré que le poids moyen des fruits et le nombre de fruits par plant sont corrélés négativement. En effet, les lignées qui ont obtenu un faible nombre de fruits par plant ont eu un poids moyen de fruits plus élevé. Par contre, la corrélation entre le nombre total de fruits récoltés et le poids total de fruits a été positive. Par ailleurs, une forte variabilité a été notée entre les lignées par rapport à ces composantes du rendement. Cette variabilité observée pourrait s'expliquer par la différence observée au niveau de l'état du développement végétatif de ces lignées. Les lignées chez lesquelles le plus grand nombre de fruits a été enregistré, ont présenté le meilleur développement végétatif. Ces résultats corroborent ceux de Lannes et al. (2007), Moreno et al. (2010) et Seleshi et al. (2011) qui ont montré des corrélations positives entre le développement végétatif et le nombre puis le poids de fruits chez le piment, une plante de la même famille botanique que la tomate.

Les résultats relatifs aux rendements (potentiel et net) ont indiqué que la lignée TM44/14EW (47,13 t/ha) a été la plus performante. Les lignées TMA97 (21,33 t/ha), TM46/15BF (20,96 t/ha), PETOM (20,23 t/ha), LINDO (19,26 t/ha), TM41/14EW $(16,53 \mathrm{t} / \mathrm{ha})$, TM39/14EW $(13,8 \mathrm{t} / \mathrm{ha})$ et TM48/04A (12,33 t/ha) ont obtenu les plus faibles rendements. D'une façon générale, le rendement de chaque culture dépendrait des caractéristiques du milieu naturel, du matériel végétal utilisé, de sa provenance et des techniques de production appliquées. La diversité de ces facteurs traduirait la grande variabilité des rendements produits par les lignées de tomate. Ces résultats ont indiqué également que la lignée TM44/14EW introduite peut s'adapter à différentes zones pédoclimatiques et assurer des rendements significatifs. Pour Senan et al. (2007), si une différence est observée entre les variétés au cours d'une même saison, elle est due à la variété seule. Il a été noté chez les lignées telles que TM16/04A, TM39/14EW et LINDO, un taux de fruits avariés très élevé. Ces lignées semblent plus sensibles aux nuisibles (insectes et maladies) qui ont occasionné la pourriture d'un nombre élevé de fruits. Les autres lignées, particulièrement celles originaires du Burkina Faso et une en provenance de la Norvège (TM43/14EW) ont produit les taux d'avarie les plus faibles. Ces dernières pourraient être jugées tolérantes aux maladies parmi les lignées utilisées dans cette étude.

Concernant la fermeté, une variabilité a été observée entre les lignées indépendamment de leur origine. Les fruits les plus fermes ont été ceux des lignées TM43/14EW et TM44/14EW ; et les moins fermes ont été ceux des lignées TM17/04A, TM39/14EW, TM41/14EW et TM48/04A. Cette différence dans la fermeté résulterait de facteurs génétiques propres à chaque lignée. En effet, la matrice de Pearson a révélé une forte corrélation entre la fermeté et les composantes du rendement dont le nombre de fruits par plant et le poids moyen du fruit. Cette corrélation a également montré que la fermeté des fruits augmente avec le nombre de fruits par plant. Par contre, elle diminue quand le poids moyen du fruit augmente. Ces observations peuvent s'expliquer par le fait que lorsque la plante porte peu de fruits, chaque fruit a assez d'espace pour augmenter de volume. Cette augmentation de volume mobiliserait beaucoup d'eau dans le fruit. Et donc, plus le fruit est riche en eau et moins ferme il est. D'après les résultats obtenus, il 
semble que le facteur qui a le plus influencé la fermeté des fruits a été le poids moyen du fruit. Chez la tomate, l'on pourrait avancer que, quand le nombre de fruits par plant est élevé, le poids moyen du fruit diminue et les fruits sont plus fermes.

L'analyse en classification hiérarchique ascendante (CHA) effectuée sur l'ensemble des variables agromorphologiques des lignées de tomate a montré que sur les quatorze descripteurs adoptés, huit se sont révélés être les plus discriminants suivant les valeurs de la statistique de Fisher (F) supérieures à 10. Ce sont l'état végétatif des plants, le délai de floraison, le nombre total de fruits, le nombre de fruits par plant, les rendements potentiel et net puis la fermeté des fruits. L'évaluation agromorphologique des lignées de tomate a ainsi mis en évidence l'existence de plusieurs caractères discriminants. Cela traduirait l'existence d'une grande diversité morphologique au sein de la collection étudiée.

\section{Conclusion}

$\mathrm{Ce}$ travail avait pour objectif principal d'évaluer les performances agronomiques de quinze (15) lignées de tomate. Au terme de cette étude, il ressort qu'au champ, l'état et le développement végétatif ont été différents chez les lignées. En effet, ce sont les lignées TM16/04A, TM43/14EW et TM44/14EW qui ont donné le meilleur état végétatif. Pour la hauteur des plants, c'est la lignée TM41/14EW qui a été la plus performante. En ce qui concerne les stades phénologiques, notamment le délai de floraison, seules les lignées TM16/04A, TM17/04A, TM43/14EW, TM44/14EW, TMA97, LINDO et PETOM ont été précoces. Relativement aux rendements, il a été noté une importante variabilité entre les lignées; particulièrement pour le rendement net, le nombre de fruits par plant et le poids moyen du fruit. La lignée TM44/14EW avec 47,13 t/ha a été la plus productive; tandis que les lignées TMA97 (21,33 t/ha), TM46/15BF (20,96 t/ha), PETOM (20,23 t/ha), LINDO (19,26 t/ha), TM41/14EW $(16,53 \mathrm{t} / \mathrm{ha})$, TM39/14EW $(13,8 \mathrm{t} / \mathrm{ha})$ et
TM48/04A (12,33 t/ha) ont été les moins performantes. Les fruits les plus fermes ont été enregistrés chez les lignées plus productives. Deux des lignées originaires de la Norvège, TM43/14EW et TM44/14EW ont obtenu les fruits les plus fermes. Tandis que quatre autres lignées introduites de Taïwan et de la Norvège, en l'occurrence TM17/04A, TM39/14EW, TM41/14EW et TM48/04A, ont eu les fruits les moins fermes. A l'issue de cette étude, il est à noter que les objectifs ont été atteints car des lignées à hauts rendements et qui s'adaptent bien aux conditions climatiques de la Côte d'Ivoire ont été identifiées.

En conséquence, la confirmation en milieu paysan de lignées telles que TM40/14EW, TM43/14EW et TM44/14EW devrait être envisagée.

\section{CONFLIT D'INTERETS}

Les auteurs ne déclarent aucun conflit d'intérêts.

\section{CONTRIBUTIONS DES AUTEURS}

NDC était l'investigateur principal ; LF et MFDPN ont contribué à l'amélioration du manuscrit ; BD a effectué les observations et les relevés.

\section{REMERCIEMENTS}

Nos remerciements à l'endroit des autorités du Centre National de Recherche Agronomique (CNRA); notamment celles de la Station de Recherche sur les Cultures Vivrières à Bouaké pour avoir mis à notre disposition des parcelles pour la mise en place de l'expérimentation. Nos remerciements également à Mme GOULIVAS Aude Viviane anciennement chargée des Cultures maraîchères au Fonds Interprofessionnel pour la Recherche et le Conseil Agricole (FIRCA) pour avoir apporté son aide pour l'introduction de lignées de tomate du Burkina. Enfin, nos remerciement à nos partenaires de East West Seed Global Orion (EWSGO) en Norvège et de Asian Vegetable Research and Development Center (AVRDC) en Taiwan 
pour les lignées de tomate qu'ils ont bien voulu nous céder.

\section{REFERENCES}

Daniel CC, Alphonse A, Jacques BA, Romaric EK, Ulrich GK, Elvis JNA. 2012. Inventaire préliminaire de l'entomofaune des champs de tomates (Lycopersicon esculentum Mill) dans la Commune de Djakotomey au Bénin. Int. J. Biol. Chem. Sci., 6(4): 1798-1804. DOI:

http://dx.doi.org/10.4314/ijbcs.v6i4.34

FAO. 2014. Food and Agriculture Organization of the United Nations, Statistics Division, http://www.fao.org/faostat/fr/\#data/QC (visité le 22/11/2017).

Fondio L, Djidji AH, N'gbesso MFDP, Koné D. 2013. Evaluation de neuf variétés de tomate (Solanum lycopersicum L.) par rapport au flétrissement bactérien et à la productivité. Int. J. Biol. Chem. Sci., 7(3): $\quad$ 1078-1086. DOI: http://dx.doi.org/10.4314/ijbcs.v7i3.15

Fondio L., N'zi JC, Kobenan K. 2015. Comportement agronomique et sanitaire de nouvelles lignées de piment dans le Sud de la Côte d'Ivoire. Journal of Applied Biosciences, 92: 8594-8609. DOI:

http://dx.doi.org/10.4314/jab.v92i1.4

Gwennaël B. 2013. Gènes architectes et sexualité chez les plantes: À la découverte des fleurs de tomate stamenless. Revue des Questions Scientifiques, 184(3): 351-372

Ignace $\mathrm{S}$, Moumouni $\mathrm{K}$, Constantin $\mathrm{D}$, Lamousssa PO, Valérie BEJTB, Adama $\mathrm{H}$, Charlemagne G, Eloi P, Roger HC N. 2015. Etude de l'influence des modes de transformation sur les teneurs en lycopène de quatre variétés de tomates de la région du nord du Burkina Faso. Int. J. Biol. Chem. Sci., 9(1): $\quad 362-370 . \quad$ DOI: http://dx.doi.org/10.4314/ijbcs.v9i1.31

INRA.

2010.

Légumes.

Ephytia.inra.fr/tomate/importance économique. Consulté le 07/03/2018.

Jean-Claude N, Christophe K, Assanvo SPN. 2019. Influence de quelques paramètres climatiques sur les effectifs de Bemisia tabaci sur la tomate (Solanum lycopersicum L.). Int. J. Biol. Chem. Sci., 13(1): 338-352. DOI: https://dx.doi.org/10.4314/ijbcs.v13i1.27 FAO 2007. FAO statistic. www.faostat.fao.org/site/612/default.asp x\#ancor (consulté le 11/ 11/ 2017).

Lannes SD, Finger FL, Schuelter AR, Casali VWD. 2007. Growth and quality of Brazilian accessions of Capsicum chinense fruits. Scientia Horticulturea, 112: 266- $270 . \quad$ DOI: doi:10.1016/j.scienta.2006.12.029

Martine D, David LE, Athanasios PP. 2008. Tomato (Solanum lycopersicum) health components: from the seed to the consumer. Phytochem Rev, 7:231-250. DOI: 10.1007/s11101-007-9085-x

Moreno MM, Moreno C, Villena J, Mancebo I. 2010. Agro-morphological characterization of 16 traditional pepper (Capsicum annuum L.) cultivars from Castilla-La Mancha (central Spain). International Symposium on Plant Genetic Resources, 2: 551-557. DOI: https://doi.org/10.17660/ActaHortic.201 1.918 .70

Muhammad D, Muhammad Q, Maray A. 2007. Effect of different level of N, P and $\mathrm{K}$. The Growth and Yield of Cabbage. Journal of Agricultural Research, 45(2): 51-68.

Musas NN. 2012. Valorisation agronomique des bio déchets et gestion de la fertilité des sols en agriculture urbaine et périurbaine : Effets des doses croissantes des engrais minéraux, des fèces humains et de leur combinaison sur la production de l'oignon (Allium cepa) et de l'épinard 
(Spinacia oleracea). Mémoire de fin d'études, faculté des sciences agronomiques, Unilu, 43 Pages.

N'Zi JC, Kouamé C, N'Guettia ASP, Fondio L, Djidji AH, Sangaré A. 2010. Evolution des populations de Bemisia tabaci Genn. Selon les variétés de tomate (Solanum lycopersicum L.) au centre de la Côte d'Ivoire. Sciences \& Nature, 7(1): $31-40$

Ojetayo AE, Olaniyi JO, Akanbi WB, Olabiyi TI. 2011. Effect of fertilizer types on nutritional of two cabbage varieties before and after storage. Journal of Applied Biosciences, 48: 3322 - 3330

Seleshi D. 2011. Evaluation of Elite Hot Pepper Varieties (Capsicum species) for growth, dry pod yield and quality under Jimma condition, South West Ethiopia. Master's thesis of Science in Horticulture (Vegetable Science). School of Graduate Studies, College of Agriculture and Veterinary Medicine: Jimma University. 73 Pages.

Senan S, Mamadou D, Daouda D, Tschannen A, Olivier G. 2007. Performance de six cultivars de tomate Lycopersicon esculentum Mill. Contre la jaunisse en cuillère des feuilles, le flétrissement bactérien et les nématodes à galles. Sciences \& Nature, 4(2): 123-130.

Ward JH. 1963. Hierarchical grouping to optimize an objective function. Journal of the American Statistical Association, 58: $236-244$.

Willcox JK, Catignani GL, Lazarus S. 2003. Tomatoes and cardiovascular health. Critical Review in food Science and Nutrition, 43(1): 1- 18. DOI: http://dx.doi.org/10.1080/104086903908 26437. 